\title{
La filière nucléaire thorium-uranium revisitée
}

\author{
Sylvain David ${ }^{1}$, Élisabeth Huffer ${ }^{2}$ et Hervé Nifenecker ${ }^{2}$ \\ Commission énergie de la Société Française de Physique \\ 1. Institut de Physique Nucléaire d'Orsay \\ 2. Laboratoire de Physique Subatomique et de Cosmologie (IN2P3, Grenoble)
}

\begin{abstract}
Une filière nucléaire thorium-uranium dans laquelle le noyau fissile principal est l'uranium 233, dont la régénération par capture neutronique est assurée par le thorium 232, présente des avantages potentiels importants par rapport à la filière actuelle uranium-plutonium : en particulier, une moindre production de déchets de haute activité et vie longue, et une prolifération nucléaire plus difficile. On présente rapidement les types de réacteurs envisagés pour mettre cette filière en œuvre. On montre enfin qu'une stratégie utilisant les réacteurs à eau pressurisée actuels, avec un combustible oxyde mixte de thorium et de plutonium, permettrait de constituer le stock initial d'uranium 233.
\end{abstract}

\section{Introduction}

Les réacteurs actuels, qui brûlent essentiellement l'uranium 235 , n'utilisent que de l'ordre de $1 \%$ de l'uranium naturel' ${ }^{1}$. De ce fait, les réserves d'uranium ne sont suffisantes que pour une durée estimée de l'ordre du siècle, cette durée dépendant du nombre de réacteurs en fonction dans le monde et du prix accepté pour l'uranium naturel. Dès les années 50 , cette relative faiblesse des réserves conduisit les physiciens des réacteurs à proposer le développement de filières surgénératrices dans lesquelles le noyau fissile principal n'est plus l'uranium 235 mais soit le plutonium 239, soit l'uranium 233. En effet, la fission de ces noyaux est accompagnée de l'émission d'un nombre suffisant de neutrons pour en assurer la régénération par capture neutronique dans l'uranium 238 et le thorium 232 respectivement. Ni l'uranium 233, ni le plutonium 239 ne sont présents en quantités significatives dans la nature. II faut donc les produire avant de pouvoir réaliser des réacteurs les utilisant. Le plutonium est produit automatiquement dans la quasi-totalité des réacteurs en fonctionnement dans le monde, qui utilisent des combustibles à base d'uranium. La disponibilité de quantités importantes de plutonium conduisit au développement de la filière dite " uranium-plutonium ", concrétisée en France par les réacteurs surgénérateurs (ou "breeders") Phénix et Super-Phénix. Au contraire, les quantités disponibles d'uranium 233 restaient confidentielles, incapables de permettre un développement rapide d'une filière «thorium-uranium ».

Il apparaît aujourd'hui que le rythme de croissance du nucléaire dans le monde n'exige pas un développement rapide des filières surgénératrices. II est donc possible, comme nous le montrerons, de constituer un stock d'uranium 233 qui pourrait, à terme, permettre le développement d'une filière " thorium-uranium ». Dans cet article, nous montrons qu'une telle filière présenterait des avantages sur plusieurs points importants, en particulier en ce qui concerne la gestion des déchets radioactifs et les risques de prolifération nucléaire. Nous présenterons rapidement les types de réacteurs envisagés pour la mettre en œuvre et une stratégie utilisant les réacteurs actuels pour constituer le stock initial d'uranium 233.

\section{Rôle des chercheurs CNRS et enseignants-chercheurs dans les études sur l'énergie nucléaire}

D'une façon générale, la loi du 30 décembre 1991 a conduit un nombre significatif d'enseignants-chercheurs et de cher-

1. L'uranium naturel contient $0,7 \%$ d'uranium 235 et $99,3 \%$ d'uranium 238. Les réacteurs français de type REP utilisent un uranium faiblement enrichi à $3,5 \%$ d'uranium 235. Les fissions proviennent à $60 \%$ de l'uranium 235 et pour le reste, essentiellement et indirectement, de l'uranium 238 en passant par le plutonium 239 résultant de captures neutroniques sur l'uranium 238 . cheurs à s'investir dans des études sur la gestion des déchets nucléaires, en particulier à travers des groupements de recherche comme PRACTIS, FORPRO, MOMAS, PARIS, GEDEON (GEDEPEON). En particulier, le CNRS a acquis une expertise de haut niveau dans les domaines du calcul de réacteurs, dans l'étude des cycles de combustible et dans la mise au point de scénarios de déploiement de systèmes nucléaires de production d'électricité. De ce fait, les chercheurs et enseignants-chercheurs ont développé des approches du nucléaire du futur qui, sans être contradictoires avec celles développées par les entreprises et organismes chargés de mettre en place la politique nucléaire française (CEA, EDF, AREVA), en sont complémentaires. II a semblé utile à la SFP que certaines de ces approches soient portées à la connaissance du public dans le cadre des Débats Publics sur la gestion des déchets nucléaires et sur le futur réacteur EPR. La contribution présente est consacrée au cycle du thorium et à la filière nucléaire thorium-uranium.

\section{Les avantages du cycle thorium}

Les réacteurs nucléaires surgénérateurs comme SuperPhénix font appel au cycle uranium 238-plutonium 239. Dans ce cycle, le plutonium dont la fission produit l'énergie du réacteur est remplacé par du nouveau plutonium obtenu grâce à la capture d'un neutron par l'uranium 238 :

$$
{ }_{92}^{238} \mathrm{U}+\mathrm{n} \rightarrow{ }_{92}^{239} \mathrm{U} \rightarrow{ }_{93}^{239} \mathrm{~Np}+\mathrm{e}^{-} \rightarrow{ }_{94}^{239} \mathrm{Pu}+2 \mathrm{e}^{-}
$$

Dans le cycle thorium 232-uranium 233, le thorium 232 joue le rôle de l'uranium 238 et l'uranium 233 celui du plutonium 239 :

$$
{ }_{90}^{232} \mathrm{Th}+\mathrm{n} \rightarrow{ }_{90}^{233} \mathrm{Th} \rightarrow{ }_{91}^{233} \mathrm{~Pa}+\mathrm{e}^{-} \rightarrow{ }_{92}^{233} \mathrm{U}+2 \mathrm{e}^{-}
$$

neutrons disponibles pour la surgénération

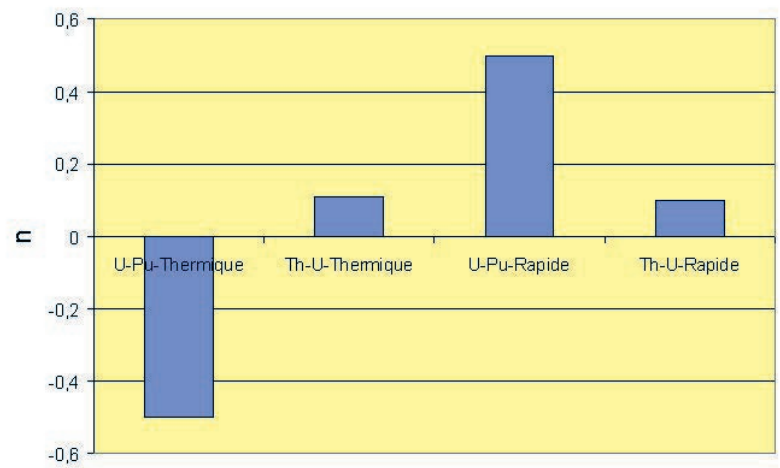

Figure 1. Valeur du nombre de neutrons disponibles " $n$ » pour la surgénération dans les systèmes uranium-plutonium et thorium-uranium, pour des spectres de neutrons thermiques et rapides. Les valeurs négatives signifient que la régénération est impossible. 
Comme on peut le voir sur la figure 1 , alors que le cycle uranium 238-plutonium 239 exige, pour fonctionner durablement, que les neutrons soient rapides, le cycle thorium 232-uranium 233 fonctionne aussi bien avec des neutrons lents qu'avec des neutrons rapides.

\section{Un faible inventaire initial}

La probabilité relative de fission des noyaux fissiles $\left({ }^{239} \mathrm{Pu}\right.$ et $\left.{ }^{233} \mathrm{U}\right)$, comparée à celle de simple capture des neutrons par les noyaux fertiles (238 $\mathrm{U}$ et ${ }^{232} \mathrm{Th}$ ), est plus grande pour les neutrons lents que pour les neutrons rapides. De ce fait, les quantités de noyaux fissiles nécessaires au fonctionnement de réacteurs à neutrons lents sont généralement plus faibles que celles nécessaires au fonctionnement des réacteurs à neutrons rapides. C'est ainsi que, dans le cas du cycle uranium 238-plutonium 239, la quantité de plutonium 239 nécessaire au fonctionnement d'un surgénérateur à neutrons rapides d'une puissance thermique de $3 \mathrm{GW}$ est, typiquement, de l'ordre de 7 à 14 tonnes $^{2}$ (selon les quantités immobilisées dans le circuit de retraitement, l'inventaire du cœur proprement dit étant de l'ordre de 7 tonnes). Dans le cas du cycle thorium 232-uranium 233, la quantité d'uranium 233 qui est nécessaire au fonctionnement d'un surgénérateur à neutrons lents de même puissance ne nécessite que 1,5 à 3 tonnes ${ }^{3}$. II s'ensuit que la fabrication de la première charge en uranium 233 d'un surgénérateur du cycle thorium 232-uranium 233, grâce à l'irradiation de thorium 232 dans un réacteur " classique ", prendrait environ quatre fois moins de temps que celle de la première charge d'un surgénérateur du cycle uranium 238-plutonium 239 obtenue par irradiation de l'uranium 238 dans un réacteur du même type.

\section{Des déchets moins radioactifs}

Au-delà de quelques siècles, la radiotoxicité des déchets nucléaires est dominée par celle des noyaux lourds $(Z>92)$ radioactifs alpha produits par des captures successives de neutrons par les noyaux lourds du combustible nucléaire. Dans le cas des surgénérateurs utilisant le cycle uranium-plutonium, ces deux derniers éléments sont, en principe, com-

\section{radiotoxicité $R(t)$ des rejets d'actinides à l'équilibre}

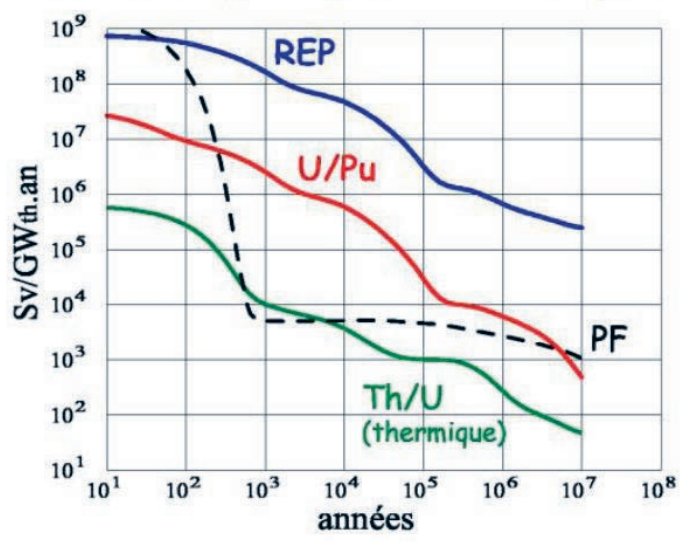

Figure 2. Évolution temporelle de la radiotoxicité des déchets de haute activité pour différents cycles, par unité d'énergie extraite du réacteur (gigawatts thermiques $x$ an). Les déchets nucléaires résultent d'une part des actinides rejetés au cours du retraitement, dont la quantité dépend du type de réacteur, et d'autre part des produits de fission (PF) dont la quantité ne dépend que de l'énergie extraite. "À l'équilibre " signifie que, pour les réacteurs à cycle fermé (RNR U/Pu et Th/U), on est dans la situation où les quantités d'actinides mineurs produits et détruits dans le réacteur sont égales.

2. Dans un surgénérateur uranium-plutonium, la charge initiale en plutonium représente entre 15 et $18 \%$ de celle en uranium.

3. Dans un surgénérateur thorium-uranium, la charge initiale en uranium 233 représente environ $1,5 \%$ de celle en thorium. plètement utilisés après retraitement, et ne concourent que très peu (pertes de retraitement) à la radiotoxicité des déchets qui est, de ce fait, deux ordres de grandeur plus faible que dans le cas des réacteurs $\mathrm{REP}^{4}$ classiques, comme on peut le voir sur la figure 2 .

En d'autres termes, à production d'énergie égale, les besoins de stockage géologique seront près de 100 fois plus faibles pour un ensemble de surgénérateurs uranium-plutonium que dans la pratique actuelle. L'utilisation du cycle thorium-uranium est encore plus favorable de ce point de vue car, le thorium 232 ayant un nombre de masse de six unités inférieur à celui de l'uranium 238, la production des actinides mineurs (neptunium, américium, curium), qui sont à l'origine de l'essentiel de la radiotoxicité des déchets du cycle uranium-plutonium, est fortement réduite. La figure 2 montre que la radiotoxicité des déchets du cycle thorium-uranium est beaucoup plus faible que celle du cycle uranium-plutonium pendant les premiers 10000 ans. En particulier, la faiblesse de la radioactivité de ce cycle au début du stockage permettrait de grandes économies sur la dimension et donc sur le coût de celui-ci ${ }^{5}$.

\section{Un combustible peu proliférant}

La disponibilité d'un noyau fissile de faible activité gamma est indispensable pour la fabrication d'une arme nucléaire. En effet, la présence d'une forte activité gamma nécessite que les opérateurs soient protégés par d'importantes épaisseurs de plomb ou de verre au plomb. L'uranium 235, obtenu grâce aux usines de séparation isotopique, n'a qu'une faible activité gamma, tout comme le plutonium extrait des combustibles irradiés. La production d'uranium 233 est, en général, accompagnée de celle d'uranium 232 de période 70 ans. Les désintégrations alpha successives de l'uranium 232 conduisent au thallium 208, dont la désintégration s'accompagne de l'émission d'un rayonnement gamma de 2,6 MeV extrêmement pénétrant. La fabrication d'une arme utilisant de l'uranium 233 contaminé par de l'uranium 232 est donc pratiquement impossible. II est vrai que cet avantage à l'égard de la prolifération se paye par la complexité de la fabrication du combustible des réacteurs, dont toutes les étapes doivent être robotisées ou, au moins, se faire à l'abri de lourdes protections.

\section{Des réserves importantes}

Les réserves terrestres de thorium sont estimées à environ quatre fois celles d'uranium. En particulier, des gisements très riches sont abondants en Inde, au Brésil et à Madagascar. Par contre, le thorium est très peu soluble dans l'eau, ce qui exclut son extraction de l'eau de mer, au contraire de celle de l'uranium. II y a lieu de souligner, toutefois, que la technologie des réacteurs surgénérateurs, très économe en combustible, permet l'exploitation rentable de minerais de faible teneur et garantirait la disponibilité de combustibles pour plusieurs milliers d'années, aussi bien pour les surgénérateurs au thorium que pour ceux à l'uranium. La plus grande abondance du thorium n'est donc pas un élément décisif.

\section{Quels réacteurs pour mettre en œuvre le cycle thorium?}

Dans le cycle thorium-uranium, la surgénération peut être obtenue aussi bien avec des neutrons rapides qu'avec des neutrons lents. Avec les neutrons rapides, elle est plus difficile que pour le cycle uranium-plutonium : elle exige des inven-

4. REP : réacteurs à eau pressurisée, les seuls utilisés en France. Les REB (réacteurs à eau bouillante) ont des caractéristiques similaires en ce qui concerne l'utilisation du combustible.

5. Ceci sans préjudice de la nécessité de gérer les stocks actuels et ceux produits par la prochaine génération d'EPR. 


\begin{tabular}{|c|c|c|c|c|c|c|}
\hline noyau & $\begin{array}{l}\text { chargement } \\
\text { (kg/8TWhe) }\end{array}$ & $\begin{array}{c}\text { Production MOX } \\
\text { (kg/8TWhe) }\end{array}$ & $\begin{array}{l}\text { Production } \\
\text { MOX Th }\end{array}$ & $\begin{array}{c}\text { facteurs de } \\
\text { qualité }\end{array}$ & $\begin{array}{l}\text { qualité Pu } \\
\text { MOX }\end{array}$ & $\begin{array}{l}\text { qualité Pu } \\
\text { MOX Th }\end{array}$ \\
\hline${ }^{233} \mathrm{U}$ & 0 & 0 & 278,72 & & & \\
\hline${ }^{239} \mathrm{Pu}$ & 1008 & -500 & -650 & ${ }^{239} \mathrm{Pu} / \mathrm{Am}$ & 3,65467626 & 3,00840336 \\
\hline Pu total & 1900 & -544 & -800 & $\begin{array}{c}{ }^{239} \mathrm{Pu} /(\mathrm{Pu} \text { total } \\
-239 \mathrm{Pu}+\mathrm{Am})\end{array}$ & 0,51469098 & 0,41579559 \\
\hline A.M. & 0 & 139 & 119 & & & \\
\hline
\end{tabular}

taires initiaux élevés en uranium 233, de l'ordre de 5 tonnes pour un réacteur de $3 \mathrm{GW}$ thermiques. À cet inventaire dans le cœur, il faut ajouter l'uranium 233 présent dans le cycle du combustible (dans l'usine de retraitement et en attente de retraitement). II s'ensuit que les temps de doublement ${ }^{6}$ deviennent très grands. En pratique, les réacteurs thoriumuranium à neutrons rapides seront des réacteurs simplement régénérateurs. Ils pourraient être du même type que les réacteurs rapides utilisant le cycle uranium-plutonium, par exemple avec des systèmes de refroidissement utilisant des métaux liquides (sodium ou plomb).

Les réacteurs surgénérateurs thorium-uranium à neutrons lents ne nécessitent qu'un faible inventaire d'uranium 233, de l'ordre de 1 tonne. Les temps de doublement théoriques sont équivalents à ceux des surgénérateurs uranium-plutonium à neutrons rapides. Toutefois, les produits de fission empoisonnent de façon beaucoup plus efficace les réacteurs à neutrons lents que les réacteurs à neutrons rapides. Pour conserver un temps de doublement intéressant, il est donc nécessaire de minimiser les captures dans les produits de fission et dans les autres éléments de structure ou caloporteurs. Une solution théoriquement très élégante à ce problème a été proposée dans les années 60 sous la forme de réacteurs dans lesquels le combustible est un sel fondu qui fait aussi office de caloporteur. Pour limiter les captures dans les produits de fission, il était proposé de recycler le sel en ligne, au prix d'une grande complexité du réacteur qui devient une usine chimique. L'abandon de l'objectif d'un faible temps de doublement permet d'envisager des réacteurs à sel fondu où le retraitement en ligne est très simplifié, ou bien d'autres types de réacteurs à déchargement continu comme les réacteurs à eau lourde (CANDU) ou les réacteurs à boulets refroidis au gaz. Un schéma particulièrement intéressant consisterait à compléter un parc de régénérateurs thorium-uranium par des réacteurs rapides utilisant un cœur régénérateur uranium-plutonium entouré d'une couverture en thorium qui fournirait l'excédent d'uranium 233 nécessaire à la croissance du parc.

\section{Avantages et inconvénients des combustibles MOX uranium-plutonium}

À la suite du choc pétrolier de 1973, les experts français avaient prévu un développement rapide de la production d'électricité nucléaire dans le monde, à l'image de la politique dans laquelle la France allait s'engager. Les ressources d'uranium, mal utilisées dans les réacteurs de type REP, risquaient d'être insuffisantes. Pour cette raison, la France, mettant à profit les connaissances acquises auprès du réacteur expérimental Rapsodie et du réacteur prototype Phénix (qui ont divergé respectivement en 1967 et 1973), s'engagea hardiment dans le développement des réacteurs surgénérateurs (RNR) refroidis au sodium, avec la construction de SuperPhénix (pré-décision en 1973, début de construction en 1976,

6. Le temps de doublement est le temps au bout duquel la quantité initiale d'uranium 233 (ou de plutonium mutatis mutandis) est doublée. divergence en 1985). De plus, il fut décidé de réaliser les usines de séparation du plutonium (La Hague) et de fabrication (Marcoule) de combustibles oxydes chargés en plutonium, nécessaires à la fourniture et à la fabrication des cœurs des réacteurs surgénérateurs.

Les prévisions des experts français ne se réalisèrent pas du fait, d'une part, du développement des centrales électriques à charbon favorisé par des coûts d'investissement plus faibles que ceux des centrales nucléaires, et d'autre part de l'accident de Three Mile Island qui mit fin à la construction de réacteurs aux USA. La justification économique des RNR disparut donc. En même temps, leur développement se heurtait à une virulente opposition du mouvement antinucléaire qui voyait en eux, à juste titre, une condition suffisante d'un nucléaire durable. La décision politique d'arrêter Super-Phénix, facilitée par les problèmes techniques ayant marqué les premières années du réacteur ${ }^{7}$, mit fin au programme de RNR en France, au moins à court et moyen terme. II fut alors décidé de tirer profit du considérable investissement technique, économique et humain représenté par les usines de La Hague et de Marcoule, en brûlant dans les réacteurs REP le plutonium extrait. C'est le programme «MOX », dans lequel le combustible est un oxyde mixte d'uranium et de plutonium, de composition proche de la stoechiométrie : $\left(\mathrm{U}_{1-\mathrm{x}} \mathrm{Pu}_{\mathrm{x}}\right) \mathrm{O}_{2}$, avec $\mathrm{x} \approx 0,05$.

Le programme MOX a des avantages indiscutables : économie d'uranium 235, diminution considérable du volume des déchets de haute activité et vie longue, conditionnement de ces derniers sous forme de verres très stables, maintien d'une compétence technique et industrielle unique au monde, qui représente un atout exceptionnel pour la France dans la perspective d'un redéveloppement du nucléaire dans le monde.

Mais le programme MOX a aussi des inconvénients. Les combustibles MOX irradiés sont entreposés dans la perspective d'utiliser le plutonium qu'ils contiennent encore dans des réacteurs du futur. Mais le plutonium ainsi entreposé est de moindre qualité et quantité que celui présent dans les combustibles "UOX " 8 avant retraitement. La pratique du MOX nuit donc à la constitution du stock de combustible nécessaire au déploiement d'un parc futur de RNR. De plus, les combustibles MOX irradiés sont environ 5 fois plus radioactifs que les combustibles UOX irradiés, ce qui rend leur entreposage et surtout leur stockage éventuel en site géologique, plus difficiles.

\footnotetext{
7. Voir « Super-Phénix, pourquoi ? » par G. Vendryes, éd. Nucléon (1997) et, sur le site de la SFP

http://sfp.in2p3.fr/Debat/debat_energie/Nucleaire/Reacteur/superphenix.htm 8. Les combustibles «UOX " sont initialement composés d'oxyde d'uranium $\mathrm{UO}_{2}$ (de structure c.f.c. type $\mathrm{CaF}_{2}$ ) faiblement enrichi. Après irradiation, ils contiennent des produits de fission, du plutonium et des actinides mineurs (1) plutonium et l'uranium et à insérer les autres éléments dans des matrices en verre. À mesure que les taux d'irradiation croissent, la proportion d'isotopes fissiles du plutonium (surtout le plutonium 239) diminue au profit d'isotopes non fissiles (surtout le plutonium 240), ce qui est un avantage sur le plan de la prolifération nucléaire, mais un inconvénient pour l'utilisation en réacteur.
} 


\section{Avantages du MOX thorium-plutonium}

Les combustibles MOX actuels comportent environ 5\% de plutonium pour $95 \%$ d'uranium naturel ou appauvri. Si l'irradiation tend à diminuer le stock initial de plutonium, la présence d'uranium 238 conduit à une reconstitution partielle de ce stock. Pour accélérer la disparition du plutonium, on peut remplacer l'uranium du MOX par du thorium, obtenant ainsi le MOX thorium-plutonium.

Actuellement un REP MOX uranium-plutonium incinère environ $650 \mathrm{~kg}$ de plutonium par an (pour la simplicité du raisonnement, on suppose ici un REP complètement « MOXé » alors qu'en réalité les REP ne sont «MOXés » que pour le tiers de leur chargement). Un REP MOX totalement chargé en thorium-plutonium devrait produire environ $280 \mathrm{~kg}$ d'uranium 233 par an, tout en incinérant environ $800 \mathrm{~kg}$ de plutonium. La production d'actinides mineurs serait réduite d'environ $20 \%$. Par contre, la qualité du plutonium au déchargement serait légèrement dégradée. Les résultats obtenus sont résumés dans le Tableau 1.

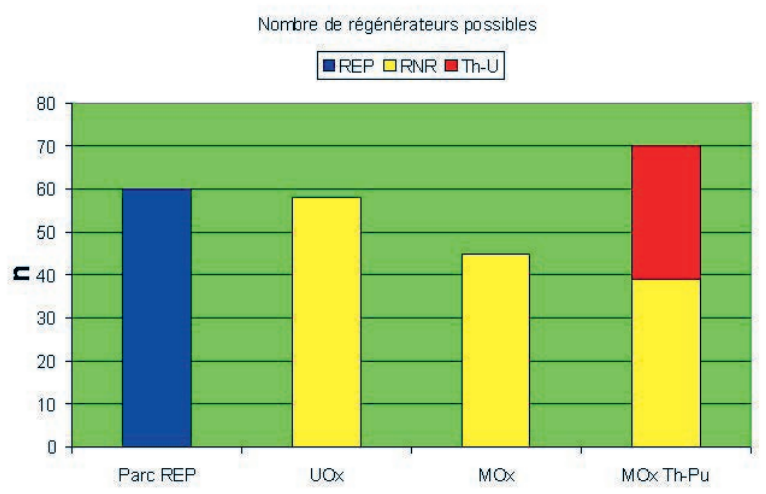

Figure 3. Nombre de surgénérateurs susceptibles d'être démarrés après 40 ans de fonctionnement d'un parc REP de 60 GW électriques. En bleu, le nombre de réacteurs REP. En jaune, le nombre de surgénérateurs uraniumplutonium pouvant être démarrés grâce au stock de plutonium produit dans le cas d'un parc non "MOXé » (UOX), d'un parc "MOXé » classique uraniumplutonium (MOX) et d'un parc "MOXé » thorium-plutonium. En rouge, le nombre de surgénérateurs thorium-uranium pouvant être démarrés dans ce dernier cas.

L'inventaire d'un surgénérateur de $1 \mathrm{GWe}$ (gigawatts électriques) à neutrons rapides uranium-plutonium est d'environ 7 tonnes. Celui d'un surgénérateur de $1 \mathrm{GWe}$ à neutrons thermiques thorium-uranium 233 serait d'environ 1,5 tonnes. L'incinération du plutonium en REP MOX uranium-plutonium fait donc disparaître $8 \%$ de l'inventaire initial d'un $\mathrm{RNR}^{9}$, alors que l'incinération en REP MOX thorium-plutonium fait apparaître environ $18 \%$ de l'inventaire d'un "breeder" thorium-uranium $233^{10}$. On n'hypothèque donc plus le développement d'un parc de surgénérateurs. De plus, le plutonium n'étant plus créé à partir de l'uranium, la quantité nette de plutonium incinéré augmenterait d'environ $150 \mathrm{~kg}$. La figure 3 illustre les considérations ci-dessus dans le cas du fonctionnement d'un parc de réacteurs REP partiellement chargés en combustibles MOX thoriés (on suppose que tout le plutonium produit dans les combustibles UOX est recyclé sous forme de MOX thorié). On constate sur la figure 3 que le nombre de surgénérateurs thorium-uranium excède significativement le nombre de surgénérateurs uranium-plutonium « perdus ", du fait de l'incinération du plutonium. Rappelons (voir l'introduction) qu'une des raisons qui a disqualifié les surgénérateurs thorium-uranium par rapport aux surgénérateurs uranium-plutonium était précisément, l'absence d'un stock d'uranium 233 alors que les réacteurs REP, REB, à eau lourde et à modérateur graphite produisaient de grandes quantités de plutonium. L'utilisation de combustibles MOX thorium-plutonium changerait complètement le panorama. De plus, en l'absence d'un programme surgénérateur, l'uranium 233 pourrait être brûlé dans des réacteurs standard sans production importante de plutonium ou d'actinides mineurs.

\section{Conclusion}

II serait regrettable que des orientations trop hâtivement décidées et en l'absence de véritable débat scientifique et technique concernant les réacteurs et cycles du futur, soient prises sous prétexte que d'autres orientations seraient trop éloignées des pratiques actuelles. II n'y a pas de véritable urgence à définir les systèmes du futur et la période se prête donc à une réflexion approfondie et ouverte. Certes, les études « papier » ne suffisent pas et il est nécessaire que des maquettes de petite dimension de réacteurs prometteurs soient réalisées dans le cadre de collaborations internationales. C'est ainsi qu'il serait très intéressant de reprendre le programme sur les réacteurs à sel fondu initié dans les années 60 par le laboratoire d'Oak Ridge aux USA, en réalisant une maquette de quelques dizaines de MW. De même, aucune raison valable ne devrait s'opposer à l'irradiation en réacteur de quelques assemblages combustibles MOX thoriés. Enfin, la France devrait proposer une collaboration au Canada et à I'Inde pour évaluer les potentialités des réacteurs CANDU visà-vis du cycle thorium-uranium 233.

\section{Pour aller plus loin}

R. Turlay (éditeur scientifique), Les déchets nucléaires, EDP-Sciences (1997), en particulier la contribution de J.P. Schapira.

P. Bonche (éditeur scientifique), L'énergie nucléaire expliquée par des physiciens, EDP-Sciences (2003)

H. Nifenecker, O. Méplan et S. David, Accelerator Driven Subcritical Reactors, IOP (2003).

A. Billebaud et H. Nifenecker, «Réacteurs Hybrides ", Techniques de l'ingénieur, BN3-235 (2005).

J.L. Bobin, E. Huffer et H. Nifenecker (éditeurs scientifiques), L'énergie de demain, EDP-Sciences (2005), en particulier la contribution de R. Brissot, et http://lpsc.in2p3.fr/gpr/french/activites.html 